\title{
Episodic Memory Retrieval, Parietal Cortex, and the Default Mode Network: Functional and Topographic Analyses
}

\author{
Carlo Sestieri, ${ }^{1,4,5}$ Maurizio Corbetta, ${ }^{1,2,3,4,5}$ Gian Luca Romani, ${ }^{4,5}$ and Gordon L. Shulman ${ }^{1}$ \\ Departments of ${ }^{1}$ Neurology, ${ }^{2}$ Radiology, and ${ }^{3}$ Anatomy and Neurobiology, Washington University School of Medicine, St. Louis, Missouri 63110, and \\ ${ }^{4}$ Department of Neuroscience and Imaging, Gabriele d'Annunzio University, and ${ }^{5}$ Institute for Advanced Biomedical Technologies, Gabriele d'Annunzio \\ University Foundation, 66013 Chieti, Italy
}

The default mode network (DMN) is often considered a functionally homogeneous system that is broadly associated with internally directed cognition (e.g., episodic memory, theory of mind, self-evaluation). However, few studies have examined how this network interacts with other networks during putative "default" processes such as episodic memory retrieval. Using functional magnetic resonance imaging, we investigated the topography and response profile of human parietal regions inside and outside the DMN, independently defined using task-evoked deactivations and resting-state functional connectivity, during episodic memory retrieval. Memory retrieval activated posterior nodes of the DMN, particularly the angular gyrus, but also more anterior and dorsal parietal regions that were anatomically separate from the DMN. The two sets of parietal regions showed different resting-state functional connectivity and response profiles. During memory retrieval, responses in DMN regions peaked sooner than non-DMN regions, which in turn showed responses that were sustained until a final memory judgment was reached. Moreover, a parahippocampal region that showed strong resting-state connectivity with parietal DMN regions also exhibited a pattern of task-evoked activity similar to that exhibited by DMN regions. These results suggest that DMN parietal regions directly supported memory retrieval, whereas non-DMN parietal regions were more involved in postretrieval processes such as memory-based decision making. Finally, a robust functional dissociation within the DMN was observed. Whereas angular gyrus and posterior cingulate/precuneus were significantly activated during memory retrieval, an anterior DMN node in medial prefrontal cortex was strongly deactivated. This latter finding demonstrates functional heterogeneity rather than homogeneity within the DMN during episodic memory retrieval.

\section{Introduction}

The default mode network (DMN) is defined as the set of brain regions that are consistently more activated during a resting or passive baseline condition than during active, goal-directed analyses of environmental stimuli (Shulman et al., 1997; Raichle et al., 2001). A similar network shows strongly correlated activity in the resting state (Greicius et al., 2003; Greicius and Menon, 2004). Because this network is highly reproducible across multiple analysis techniques and experimental conditions, it is often described as a unitary, homogeneous system that is primarily responsible for many aspects of internally directed cognition, such as episodic memory, theory of mind, self-evaluation, and introspection (for review, see Buckner et al., 2008).

Yet the DMN may also interact with non-default regions during internally directed cognition. Cortical regions inside and outside the DMN [e.g., prefrontal cortex (PFC), intraparietal sulcus (IPS) (Buckner and Wheeler, 2001; Fletcher and Henson, 2001)]

Received June 28, 2010; revised Dec. 18, 2010; accepted Dec. 28, 2010.

This work was supported by The J. S. McDonnell Foundation and by National Institute of Mental Health Grant R01 MH-71920-06. We are grateful to Abraham Z. Snyder and Annalisa Tosoni for helpful discussions, Daniel L. Pope for technical help, and Mark McAvoy for guidance with statistical analyses.

Correspondence should be addressed to Carlo Sestieri, Gabriele D'Annunzio University, Department of Neuroscience and Imaging, Institute for Advanced Biomedical Technologies, Via dei Vestini, 33, 66013 Chieti, Italy. E-mail: c.sestieri@unich.it.

DOI:10.1523/JNEUROSCI.3335-10.2011

Copyright $\odot 2011$ the authors $\quad 0270-6474 / 11 / 314407-14 \$ 15.00 / 0$ play important roles during episodic memory retrieval. Vilberg and Rugg (2008) suggested that regions in the left angular gyrus (AG), part of the DMN, may directly support episodic retrieval through their known anatomical and functional connections with the medial temporal lobe (Rushworth et al., 2006; Vincent et al., 2006; Uddin et al., 2010), whereas more dorsal regions along the IPS may support postretrieval processes, such as memoryguided decision making. Here, we provide novel evidence concerning the functions of regions inside and outside the DMN during episodic memory retrieval.

The unitary nature of DMN activity during episodic retrieval has also been questioned. Studies focusing on network functional architecture indicate that the DMN involves interacting subsystems (Buckner et al., 2008; Uddin et al., 2009; Andrews-Hanna et al., 2010; Mayer et al., 2010), each potentially responsible for specific aspects of internally directed cognition. "Old/new" and "remember/know" effects are typically observed during episodic memory tasks in regions that overlap with posterior DMN nodes (Wagner et al., 2005), whereas the medial prefrontal cortex (mPFC) seems to be preferentially activated when the memory task involves autobiographical information (Svoboda et al., 2006; McDermott et al., 2009), suggesting that the DMN may dissociate during episodic retrieval. However, no study has shown a taskinduced dissociation of anteroposterior nodes of the DMN. Here, we provide novel evidence for this dissociation. 
In the present paper, we independently defined the DMN, based on the deactivations induced by a perceptual search task and resting-state functional connectivity analyses, and then measured task-evoked activity during a memory search task that temporally isolated memory retrieval from the response indicating the memory judgment. We observed a strong functional dissociation in the time courses of task-evoked activity between parietal regions located inside and outside the DMN. Moreover, only DMN parietal regions showed task-evoked and resting-state relationships with a parahippocampal region. These results support the hypothesis that the former regions directly support memory retrieval, whereas the latter are more involved in postretrieval processes such as memory-based decision making. Finally, whereas posterior nodes of the DMN were activated during episodic memory retrieval, the primary anterior node in mPFC was deactivated, indicating that the homogeneity of the DMN that has been observed during externally directed tasks does not hold during episodic memory retrieval.

\section{Materials and Methods}

Subjects

Nineteen healthy right-handed subjects (mean age, 26.8 years; range, 23-32 years; three males) gave informed consent in accordance with guidelines set by the Human Studies Committee of Washington University. Each participant performed a perceptual search task and an episodic memory search involving audiovisual material on different days, counterbalanced across subjects. In addition, subjects were scanned at rest for $\sim 30 \mathrm{~min}$ [resting-state functional magnetic resonance imaging (rsfMRI)]. Exclusion criteria were insufficient knowledge of the English language and familiarity with the audiovisual material used. Some results from this dataset have been recently published (Sestieri et al., 2010).

\section{Study materials}

Perceptual search task. A total of 250 sentences was generated, instructing subjects to search for a specific target that could appear at any time and location in a subsequent video clip: a colored object ("Do you see a light brown bag laying somewhere in the room?") or a specific character ("Can you detect a man standing on the street wearing red pants?”). Fifty sentences from this set served as catch trials (see below, Linear modeling). The mean length of the sentences was $12.4 \pm 1.2$ words or $59.9 \pm 3.7$ characters (spaces included). Sentences were presented in white color, arial font, within a window, covering $\sim 4.5 \times 4$ degrees of visual angle located at the center of a black display. Two hundred 12-s-long video clips were extracted from four movies directed by Robert Altman (Nashville, Short Cuts, Kansas City, and A Prairie Home Companion). Clips were in colors, covering $\sim 8.7$ degrees of visual angle on the horizontal axis and were presented with the associated soundtrack. A central white fixation cross was superimposed over the clip. The clips contained ordinary types of events involving different people in a variety of contexts. One hundred twenty video clips (target trials) contained the target specified in the preceding sentence. Targets were presented on the display for a variable amount of time across trials (mean, $1808 \mathrm{~ms}$; range, 467-2970 ms), but their duration on the screen was always within a specified interval (early, $0-4 \mathrm{~s}$; middle, $4-8 \mathrm{~s}$; late, $8-12 \mathrm{~s}$ ). Forty video clips (oddball trials) did not contain the specified target but contained a $400 \mathrm{~ms}$ duration visual effect, a transparent expanding ripple that started from the center of the clip, which could appear in one of the three time intervals specified above. Oddball trials were included in the linear modeling of the blood oxygenation level-dependent (BOLD) signal, but since they were not the focus of the present paper, they were not used for behavioral analyses and will not be discussed further. The remaining 40 video clips (nontarget trials) did not contain a specific target. Twenty additional sentences and 16 video clips were used as practice material before the actual task.

Episodic memory search task. The study material for the encoding session consisted of two $\sim 28$ min episodes from an English language television sitcom (Curb Your Enthusiasm, by Larry David; Home Box Office) depicting the events in the life of the main character. The episodes (sea- son 1 , chapters 4 and 5) contained ordinary types of events (e.g., a lunch in a restaurant, a visit to the doctor). Real life, highly contextualized episodic memory encoding has been shown to result in particularly robust episodic memory retention (Furman et al., 2007). During the retrieval phase of the study, 200 sentences were presented addressing memory for details and events across the two episodes. Sentences were presented in white color, within a window covering $\sim 4.5 \times 4$ degrees of visual angle that was located at the center of a black display. One hundred sixty sentences were used for the retrieval task while the remaining 40 sentences were used as catch trials to estimate the activations produced by sentence reading (see Materials and Methods). To promote a strong episodic memory search effort and obtain sufficiently long retrieval times, we conducted pilot experiments in which we tested accuracy and retrieval times for different kinds of questions. For the present study, we focused on three main kinds of sentences: questions about specific details of a single event ("SE") (e.g., "In Larry's office, there was a poster of a clown behind his secretary's desk"), of order between two events ("OE") (e.g., "Richard mentioned his problem with alcohol before his intimacy problem"), or of multiple events ("ME") (e.g., "Larry argued with the nurse in the waiting room more than three times"). Each question was either true or false. The mean length of a sentence was $12.1 \pm 1.9$ words or $66.4 \pm 8.3$ characters (spaces included). Twenty additional sentences served as practice material before the actual task. Occasionally, while subjects were answering a question concerning one episode, a question was presented concerning the other episode. We refer to these questions as "invalid questions." However, the BOLD responses to these questions were not the focus of the present paper, and, although they were specified in the general linear model, they will not be discussed further.

\section{Procedure}

Perceptual search task. The task structure is depicted in Figure 1a. On each trial, a sentence was presented on the screen for $4 \mathrm{~s}$ instructing subjects to search for a specific target that could appear at any time and location in a subsequent video clip. Next, a video clip, covering $\sim 8.7$ degrees of visual angle on the horizontal axis, was presented at the center of a black display for $12 \mathrm{~s}$, followed by a variable intertrial interval (ITI) $(\sim 4.1, \sim 6.2, \sim 8.3$ s) with a central red fixation cross. Three kinds of clips were presented: clips containing the target ("target"), clips containing an oddball target ("oddball"), and clips not containing any target ("nontarget"). The target could appear at any time during the video clip, resulting in variable search time across trials. The diagram in Figure 1 illustrates examples of early $(0-4 \mathrm{~s}$ after sentence offset), middle $(4-8 \mathrm{~s})$, and late $(8-12 \mathrm{~s})$ trials, representing different search times. Subjects were instructed to read the sentence and search for the specific visual target in the upcoming video clip, while maintaining central fixation. When either the target or the oddball target was detected, subjects had to press one of the two "yes" keys, depending on their confidence (high, low), as quickly as possible. If a target was not detected, they had to press one of the two "no" buttons, depending on their confidence, at the end of the clip. Response confidence was rated with the following category-key mapping: left middle finger, yes, high confidence; left index, yes, low confidence; right index, no, low confidence; right middle, no, high confidence.

Subjects knew that targets could appear only once during the clip and they were instructed to passively watch the end of the clip after a target had been detected. During "catch" trials, the sentence was immediately followed by the black display containing the red fixation cross. Responses were categorized in four classes: "hit," if one of the yes buttons was pressed during either a target or an oddball trial, within a time window starting at target onset and ending $1.5 \mathrm{~s}$ after target offset; "miss," if one of the no buttons was pressed at the end of either a target or an oddball trial; "correct rejection" (CR), if one of the no buttons was pressed at the end of a nontarget trial; "false alarm" (FA) if one of the yes buttons was pressed outside the time window during a target or oddball trial, or during a nontarget trial. A total of 10 runs, each containing 25 trials, was conducted.

The perceptual task, which requires search of a complex environment and detection of a visual target, differs from traditional visual search 

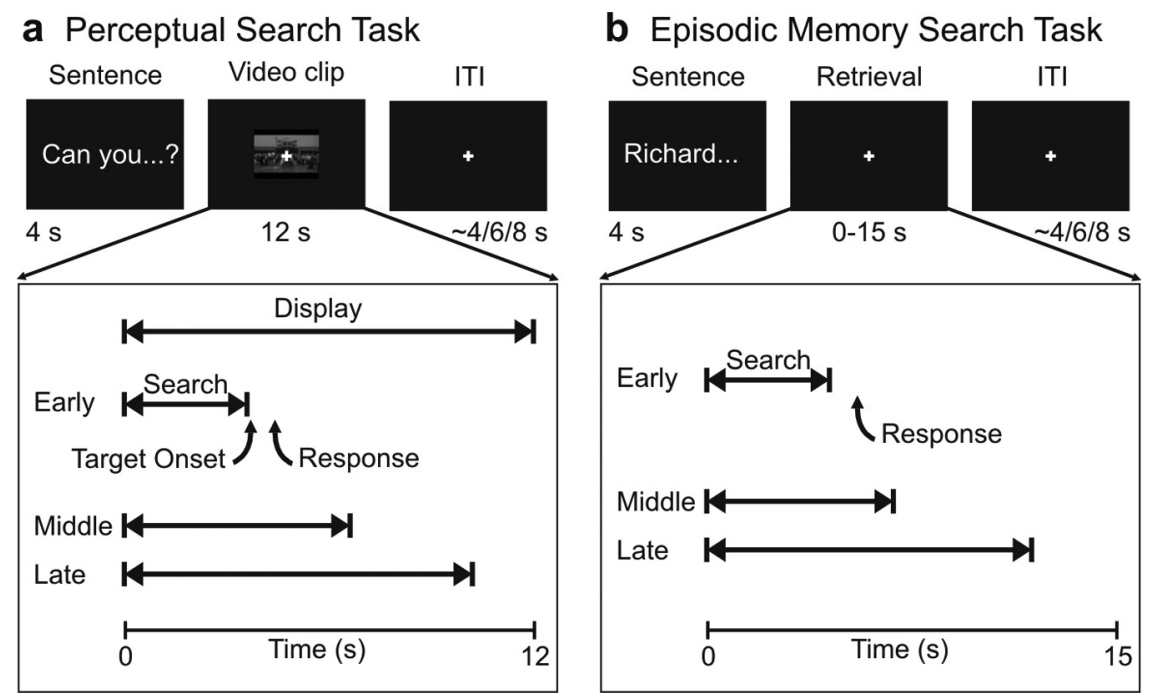

Figure 1. Perceptual search and episodic memory search tasks. $\boldsymbol{a}$, Trial structure in the perceptual search task. A sentence instructed subjects to search for a specific target (object or character) that could appear at any time in the upcoming $12 \mathrm{~s}$ video clip. Subjects searched for the target while fixating a central cross and pressed a button as soon as the target was detected. Search duration was varied (early, middle, late) by manipulating the time at which the target was presented. After display offset, a variable ITI was interposed before the onset of the next sentence. $\boldsymbol{b}$, Trial structure in the episodic memory search task. Subjects read a sentence describing a specific detail of a previously encoded episode from a TV show. They then retrieved information from episodic memory to judge the accuracy (i.e., true, false) of the sentence, which they indicated by pressing one of two buttons. Subjects were given up to $15 \mathrm{~s}$ to provide the judgment on each trial. An example of early, middle, and late search trials are provided. After subjects' response, a variable ITI was interposed before the onset of the next sentence.

paradigms (Nakayama and Silverman, 1986; Treisman, 1988). Subjects have no previous knowledge of the distracters, which vary across many dimensions, and have only a verbal description of the target. Nonetheless, this task involves visual displays similar to those involved in the memory task and requires active goal-directed analysis of environmental stimuli, which is known to deactivate the DMN.

Episodic memory search task. During the encoding session, subjects watched two episodes from an English language television sitcom. The episodes were presented in a counterbalanced order, in two different dark rooms, separated by an hour break. Subjects were instructed to watch the movie, trying to focus on it and remain concentrated throughout, since later they would be given a memory test involving the presented material. Subjects were told to avoid any specific memory strategy favoring a particular kind of memory (e.g., verbal, visual) over the others. They reported no difficulty following these instructions.

Approximately $24 \mathrm{~h}$ after the encoding session, subjects performed the fMRI retrieval session. The task structure of the episodic memory search task is depicted in Figure 1b. On each trial, a sentence addressing memory for details and events across the two episodes was presented for $4 \mathrm{~s}$ at the center of the screen. The sentence was followed by a memory search phase in which a black display with a white central fixation cross was presented. Subjects were instructed to read the sentence, wait until it disappeared, and then take the time they needed, up to $15 \mathrm{~s}$, to retrieve the specific information and provide a yes/no judgment, depending on their confidence (high, low), about the accuracy of the sentence (defined as the detection phase for consistency with the perceptual task), while maintaining central fixation. Judgments were made using four buttons with the same category-key mapping used for the perceptual task.

In this case, the source of search duration variability was the subject's decision time, reflecting the time needed to retrieve the critical information and answer the question. The diagram in Figure $1 b$ illustrates examples of early ( $0-4 \mathrm{~s}$ after sentence offset), middle ( $4-8 \mathrm{~s})$, and late (8-12 s) trials representing different search times. After the subject's response, the fixation cross turned red, indicating the onset of a variable intertrial interval $(4.1,6.2,8.3 \mathrm{~s})$ that preceded the next sentence. During catch trials, the sentence was immediately followed by the black display containing the red fixation cross. Subjects were asked not to retrieve information after catch trial sentences. Five runs of 20 trials pertaining to the episode encoded first were presented, followed by five runs pertaining to the episode encoded second.

Throughout the paper, we use the phrase memory search or memory retrieval as a proxy for a broad range of processes that involve the retrieval of episodic information guided by the behavioral goal, the organization and evaluation of the retrieved information, and the accumulation of evidence that pointed to a particular response (Moscovitch and Winocur, 1995; Badre and Wagner, 2007; Mecklinger, 2010). These processes should not be necessarily considered as organized serially, but rather as processes in cascade or continuous flow. In addition, the current task differs from many recognition tests since stimuli presented at retrieval (written sentences) do not reproduce the study material (audiovisual episodes) and are used as cues for recovering detailed memory representations. As noted by Mendelsohn et al. (2010), this type of retrieval, which they termed "cued recollection," likely involves elaborative recall processes of imagery and scene reconstruction, in addition to a general sense of familiarity.

\section{Imaging methods and preprocessing of $B O L D$ images}

Images were acquired with a Siemens Allegra 3T scanner. Structural images were obtained during the first scanning session using a sagittal magnetization-prepared rapid-acquisition gradient echo T1-weighted sequence [repetition time (TR), $1810 \mathrm{~ms}$; echo time (TE), $3.93 \mathrm{~ms}$; flip angle, $12^{\circ}$; time for inversion, $1200 \mathrm{~ms}$; voxel size, $1 \times 1 \times 1.25 \mathrm{~mm}$ ] and a T2-weighted spin-echo sequence (TR, 3800 ms; TE, 90 ms; flip angle, $\left.90^{\circ}\right)$. BOLD contrast functional images were acquired using a gradient echo echo-planar sequence (TR, $2.064 \mathrm{~ms}$; TE, $25 \mathrm{~ms}$; flip angle, $90^{\circ} ; 32$ contiguous $4 \mathrm{~mm}$ axial slices; $4 \times 4 \mathrm{~mm}$ in-plane resolution). BOLD images were motion-corrected within and between runs, corrected for across-slice timing differences, resampled into $3 \mathrm{~mm}$ isotropic voxels, and warped into a standardized atlas space (Talairach and Tournoux, 1988). Preprocessing included a whole-brain normalization that corrected for changes in overall image intensity between BOLD runs [but not between magnetic resonance (MR) frames within a run]. Importantly, since the same scaling factor was uniformly applied to all MR frames within a BOLD run, this normalization could not result in artifactual deactivations (Aguirre et al., 1998).

\section{Linear modeling}

Data were analyzed using two general linear models (GLMs). The overall approach has been described and validated in several previous studies (Ollinger et al., 2001a,b; Shulman et al., 2003, 2007; Sestieri et al., 2010). Supplemental Figure 1 (available at www.jneurosci.org as supplemental material) illustrates the linear modeling method that was used for the analysis of the perceptual and the memory task.

The "process" GLM. The aim of the first regression model was to separately estimate the BOLD signal for the different task processes that temporally overlapped in the course of a trial (Courtney et al., 1997; D'Esposito et al., 1999; Todd and Marois, 2004). For example, in the perceptual task, BOLD signals in a particular cortical region could be affected by sentence reading (sentence), by visual and auditory stimulation (display), by search for a target (search), by target detection and response (detection), or by a combination of these processes. Analogously, in the memory task, BOLD signals in a particular region could be affected by sentence reading (sentence), by search in memory (search), by responding to the question (detection), or by a combination of these processes. A multiple parameter regression model was created that specified the effects of the various task processes on the observed BOLD. The model assumed that the observed BOLD response on each trial was the 
sum of the hemodynamic responses that were generated by the above processes, and was used to identify the voxels that were activated or deactivated by each process. The assumed response for each process was generated by convolving a function representing the duration of the process (rectangle functions for sustained processes, delta functions for transient processes) with a standard hemodynamic response function (Boynton et al., 1996).

The experiment was specifically designed to enable the separation of the component processes by the process model. The sentence-reading phase was separated using a catch trial technique (Shulman et al., 1999; Ollinger et al., 2001a,b), in which on a random $20 \%$ of the trials, the trial ended after the sentence-reading phase. This technique has been validated and widely used to separate the BOLD signals to a cue from the signals to subsequent events (e.g., targets). In the current paradigm, the "cue" is represented by sentence presentation (and the associated processes such as reading), whereas the "target" is represented by memory retrieval (in the broad sense discussed above) and response. The rationale here is that, since subjects perform the same operations during catch and "full" trials, the BOLD response associated with sentence reading can be isolated and subtracted from the overall BOLD activity of full trials.

The display, search, and detection processes of the perceptual task were separated by arranging for them to have very different temporal profiles, which decorrelated the corresponding regressors in the linear model. Whereas the display duration was always $12 \mathrm{~s}$, the search duration could vary over trials from 1 to $12 \mathrm{~s}$. Similarly, whereas the display and search components were sustained processes that always began at display onset, the detection process was a transient event that randomly occurred at very different times after display onset. A total of 17 parameters was created. A sustained sentence parameter modeled the $4 \mathrm{~s}$ reading time. A sustained display parameter modeled the $12 \mathrm{~s}$ of audiovisual stimulation presented on each trial. Different versions of the search parameter specified different search outcomes. The duration of the search process, corresponding to the duration for which the display was searched, varied across trials depending on the subject's response (Shulman et al., 2007). A "Search-Hit-HC" and a "Search-Hit-LC" parameter separately coded searches on high- and low-confidence hit trials. The model assumed that the search process for these trials terminated at target onset to avoid contamination from activity associated with decision making, action planning, and action execution, processes that are associated with target detection rather than search. A "Search-Miss" and a "Search-CR" parameter separately modeled searches that lasted until the end of the clip, whereas a "Search-FA" parameter modeled search on false alarm trials until the onset of the subject's response. Finally, a "Search-withoutResponse" parameter coded trials in which subjects did not press any key. Transient BOLD responses related to the detection response phase of the trial were separately estimated. Four detection parameters ("Detection-TargetHC," "Detection-Target-LC," "Detection-Oddball-HC," "DetectionOddball-LC") modeled detection-related processes at stimulus onset separately for the two levels of confidence (high, low) and two kinds of target (target, oddball). Two miss parameters ("Miss-HC," "Miss-LC") were estimated at the time of the missed target presentation for the two levels of confidence. One "FA-Response" parameter was estimated starting at the onset of the false alarm response. Finally, a "Miss-Response" and a "CRResponse" parameter were estimated starting at display offset.

The search and detection processes in the memory task were separated by exploiting the large variability of retrieval time across trials. Specifically, whereas the search component was a sustained process that always began at cue sentence offset, the detection process was a transient event that randomly occurred at very different times after search offset. The process GLM for the memory paradigm included 11 task-related parameters. Two sustained sentence ("Sentence-Val," "Sentence-Inv") parameters modeled the $4 \mathrm{~s}$ reading time for valid or invalid questions. Five search parameters were generated according to the subject's response. To avoid contamination from activity associated with decision making, action planning, and action execution, we defined the duration of search on each trial by subtracting a constant term from the reaction time (RT) for each individual. This constant term, calculated from the perceptual task, was the mean RT across subjects from target onset (1664 ms). Four search parameters ("Search-Corr-HC," "Search-Corr-LC," "Search-Inc-
HC," "Search-Inc-LC") separately coded for searches on high and low confidence, correct and incorrect trials, whereas a fifth parameter modeled trials in which subjects did not press any key. Finally, four transient detection parameters ("Detection-Corr-HC," "Detection-Corr-LC," "Detection-Inc-HC," "Detection-Inc-HC") modeled high- and lowconfidence correct and incorrect responses, with an onset corresponding to the offset of the search process.

The "frame-by-frame" GLM. Although the previous model estimated the selective contribution of each process to the observed BOLD response, it involved assumed response functions. To examine the overall time course of BOLD activity for different types of trials (e.g., early, middle, and late correct response trials), which reflects the sum of the BOLD signal for the processes operative on those trials, a second type of GLM was created that made no assumption about the shape of the hemodynamic response. This frame-by-frame model provided an unbiased estimate of the time course for each trial type (Ollinger et al., 2001a,b), generating separate delta function regressors for each MR frame up to $\sim 30$ s after trial onset.

Two different types of frame-by-frame GLMs were created, a "between-trial" GLM and a "within-trial" GLM (Shulman et al., 1999). The between-trial GLM yielded a separate estimate of the BOLD time course for each trial type, such as sentence-reading catch trials and the different types of full trials (e.g., early, middle, and late correct response trials). Overall, time courses for eight types of trials, all starting at sentence onset, were estimated in the memory task: sentence-reading catch trials, three correct response trials (early, middle, late), three incorrect response trials (early, middle, late), and trials in which subjects did not press a key. Therefore, for example, the time course for a late correct trial indicated the time course over the entire trial starting from the onset of the sentence, and reflected the sum of the BOLD signals for all the processes operative on that trial (sentence reading, search, and detection).

To examine the time courses of BOLD activity associated with episodic memory retrieval without the contribution of the sentence-reading component, we also designed a within-trial model, in which the sentencereading phase (whether it occurred in catch trial or as part of a full trial) and the subsequent phase (search and detection) of full trials were separately estimated. Therefore, in the within model, the time courses for different trial types started at the onset of memory search, not of the sentence, and did not reflect the contribution of the preceding sentence-reading phase (Shulman et al., 1999; Ollinger et al., 2001a,b). Again, eight time course regressors were estimated: one regressor for sentence reading, three regressors for the postsentence task phase (one for each type of correct trial; early, middle, late), three regressors for the postsentence task phase (one for each type of incorrect trial; early, middle, late), and a regressor for the postsentence task phase on trials in which the subject did not press a key.

Two frame-by-frame GLMs were also created for the perceptual task. In the between-trial GLM, time courses for 13 types of trials were estimated: sentence reading, three hit-target, three hit-oddball, three miss [depending on the interval of target presentation (early, middle, late)], CR and FA trials, as well as trials in which subjects did not press a key. In the within-trial GLM, the sentence-reading phase of a trial, whether it occurred in a sentence-reading catch trial or as part of a full trial, was separately estimated with a single regressor. Again, time courses regressors for 13 types of trials were estimated in the perceptual task, like in the between-trial GLM.

\section{Resting-state functional connectivity MRI analysis for DMN definition}

We conducted six rs-fMRI runs ( $\sim 5 \mathrm{~m}$ each) in which the BOLD signal was measured while subjects maintained fixation on a central cross in an otherwise blank display. Resting-state functional connectivity MRI (rsfcMRI) assesses the temporal correlation between BOLD time series in different regions of the brain at rest, with no experimenter-imposed task. After the standard preprocessing of BOLD images described above, data were passed through an additional series of processing steps for the assessment of resting-state functional connectivity (Fox et al., 2006; Vincent et al., 2006; He et al., 2007). First, the six runs were concatenated. For each voxel, temporal filtering retained frequencies $<0.1 \mathrm{~Hz}$ and data were spatially smoothed using a $6 \mathrm{~mm}$ full-width half-maximum Gaussian blur. Several sources of spurious or regionally nonspecific variance 
were then removed by linear regression including: six parameters obtained by rigid body head motion correction, the signal averaged over the whole brain, the signal averaged over the lateral ventricles, and the signal averaged over a region centered in the deep cerebral white matter.

The DMN was defined using the following procedure: first, we identified the peaks of deactivation from the map of the perceptual searchrelated deactivation. Four peaks were selected based on their proximity to the main components of the DMN (Shulman et al., 1997; Mazoyer et al., 2001; Buckner et al., 2008): mPFC, posterior cingulate cortex (PCC), left AG, and right AG. Regions of interest (ROIs) ( $6 \mathrm{~mm}$ radius) centered on these peaks were generated and served as seeds to create functional connectivity maps in each subject.

To create voxelwise maps for each seed region, the BOLD time series from the rs-fMRI session was averaged over all voxels in the ROI, the voxelwise Pearson correlation coefficients between that seed time course and all other voxels were computed, and the Fisher $z$-transform was applied. For the group statistical analysis, a one-sample $t$ test with subject as a random effect was computed on the Fisher $z$-transformed values and the resulting group maps were Monte Carlo corrected over the brain for multiple comparisons (voxel size, $3 \times 3 \times 3 \mathrm{~mm}$; cluster size, 17 voxels; $z=3$, corresponding to $p<0.05$ ). We then created a conjunction map representing, for each voxel, the number of seeds (1-4) showing significant positive correlation with that voxel. Only voxels showing positive correlation with at least three seed ROIs were used to define the DMN.

\section{fMRI statistical analysis}

Using the process GLM, we created group voxelwise statistical maps [Monte Carlo corrected over the brain for multiple comparisons (voxel size, $3 \times 3 \times 3 \mathrm{~mm}$; cluster size, 17 voxels; $z=3$, corresponding to $p<$ $0.05)]$ in which subject was treated as a random effect. One-sample voxelwise $t$ tests were conducted to determine voxels in which a parameter was significantly different from zero. To focus on optimal task performance, voxelwise maps of perceptual and memory search-related activity were obtained using only Search-Hit-HC and Search-Corr-HC trials, respectively.

ROIs were created using a peak-search algorithm that identified peaks in the uncorrected $z$-maps and consolidated foci closer than $12 \mathrm{~mm}$ by coordinate averaging. Spherical ROIs (radius, $9 \mathrm{~mm}$ ) were formed, centered on the consolidated foci, that excluded voxels not contained in the multiple-comparison corrected $z$-map. For the creation of ROIs from the memory search activation map, an additional masking procedure was performed to ensure that all the voxels were unambiguously located inside (intra-DMN) or outside (extra-DMN) the independently defined DMN. Time courses of BOLD activity for catch trials and early, middle, and late correct trials in the memory task were obtained using both versions of the frame-by-frame GLM. One subject was excluded from this analysis since she did not provide answers in the shortest interval. Once the group-averaged time course of BOLD activity was estimated in a ROI, a simpler version of the process GLM [including only four parameters for the perceptual task (display, search, detection, and end responses) and two parameters for the memory task (search, detection)] was fit to the estimated time courses to verify both the degree to which the model described the underlying time courses (adjusted $R^{2}$ ) and the contribution of each parameter to the overall response (Betas) (see supplemental Fig. 1, available at www.jneurosci.org as supplemental material).

The latency of the peak of activity in parietal regions showing memory search-related activity was estimated using the time courses obtained with the frame-by-frame between-trial GLM. To get a robust measure of peak latency (expressed in MR frames), we calculated the weighted average of the peak frame and the frames just before and after the peak (i.e., weighting each of the three frames by the magnitude at that frame). A three-way repeated-measures ANOVA, with DMN (intra-, extra-), region (two levels), and interval (early, middle, late) as factors, assessed the presence of significant difference across parietal regions on the weighted values of peak latency for different retrieval intervals.

The voxelwise map of the BOLD response for memory detection was obtained using correct trials, collapsing over retrieval confidence. A onesample $t$ test (detection parameter vs the baseline, using the process GLM) was performed in parietal intra- and extra-DMN regions showing memory search-related activity to test whether they also exhibited a significant transient response for memory detection. Statistical comparisons between time courses of BOLD activity of different ROIs (each of the extra-DMN ROIs vs motor cortex ROI) were performed using a two-way ANOVA with region (two levels) and time (15 MR frames corresponding to the BOLD response to the whole trial) as factors.

All the search parameters (process GLM) were collapsed for the analysis of BOLD-behavior relationship in the memory task, and new ROIs were formed following the same procedure. One subject was excluded from the analysis since he did not provide a sufficient number $(<10)$ of low-confidence responses. Two-tailed paired $t$ tests were performed in the obtained ROIs to determine whether the Search-Corr-HC parameter differed from the Search-Corr-LC parameter. For one-sample and paired $t$ tests, a Bonferroni correction was used to account for the number of multiple comparisons.

\section{Additional fMRI statistical analysis}

We performed two sets of control analyses. First, we tested whether the dissociation between anterior and posterior DMN nodes held also when using a DMN definition based on a pure deactivation criterion (supplemental Fig. 4, available at www.jneurosci.org as supplemental material). Spherical ROIs (radius, $9 \mathrm{~mm}$ ) were formed, centered on the peaks of perceptual search-related deactivations used to define the DMN. Time courses of BOLD activity for catch trials and early, middle, and late trials of both the perceptual and the memory tasks were extracted from each ROI using the frame-by-frame between-trial GLM. In addition, we tested the statistical significance of the BOLD response to the memory SearchCorr-HC parameter (one-sample $t$ test vs the baseline) in each ROI using the process GLM.

Second, we tested whether the ROIs centered on the peaks of memory search-related activity were also deactivated during the perceptual search task, in accordance to a DMN definition based on deactivation criterion (supplemental Fig. 5, available at www.jneurosci.org as supplemental material). Time courses of BOLD activity for catch trials and early, middle, and late trials of both the memory and the perceptual tasks were extracted from each ROI using the frame-by-frame, between-trial GLM. Furthermore, we tested the statistical significance of the BOLD response to the perceptual Search-Hit-HC parameter (one-sample $t$ test vs the baseline) in each ROI using the process GLM.

\section{Additional $r s-f c M R I$ analyses}

To determine whether intra- and extra-DMN parietal regions belonged to different resting-state functional networks, we formed five seed ROIs ( $6 \mathrm{~mm}$ radius), centered on the peaks of memory search-related activations on the left hemisphere, corresponding to the AG, the posterior cingulate cortex-precuneus (PCC-PreCu), the anterior intraparietal sulcus (aIPS), the postcentral sulcus (PoCS), and the posterior parahippocampal gyrus (pParaHC). Corrected group voxelwise maps were created for each seed using the same procedure described above. In addition, we calculated the significance of rs-fcMRI between pairs of seed regions. For each subject, the correlation coefficient between the two regional time courses was computed and the Fisher $z$-transform was applied. A cross-correlation matrix was created showing the groupaveraged correlation coefficient of any given pair of seed regions. Significant correlation between pairs of seed regions was assessed using a onesample $t$ test on the Fisher $z$-transformed values from the individual subjects.

\section{Results}

\section{Behavior}

The accuracy of the perceptual search task was $0.69 \pm 0.1$ (oddball target excluded) and was also significantly different for highconfidence $(0.79 \pm 0.2)$ and low-confidence $(0.39 \pm 0.2)$ judgments $\left(t_{(18)}=10.72 ; p<0.001\right)$. The accuracy of the episodic memory search task was $0.73 \pm 0.1$ (chance level, 0.5 ).

Supplemental Figure $2 a$ (available at www.jneurosci.org as supplemental material) shows that the paradigm successfully varied the duration of memory retrieval over a wide range of values, 

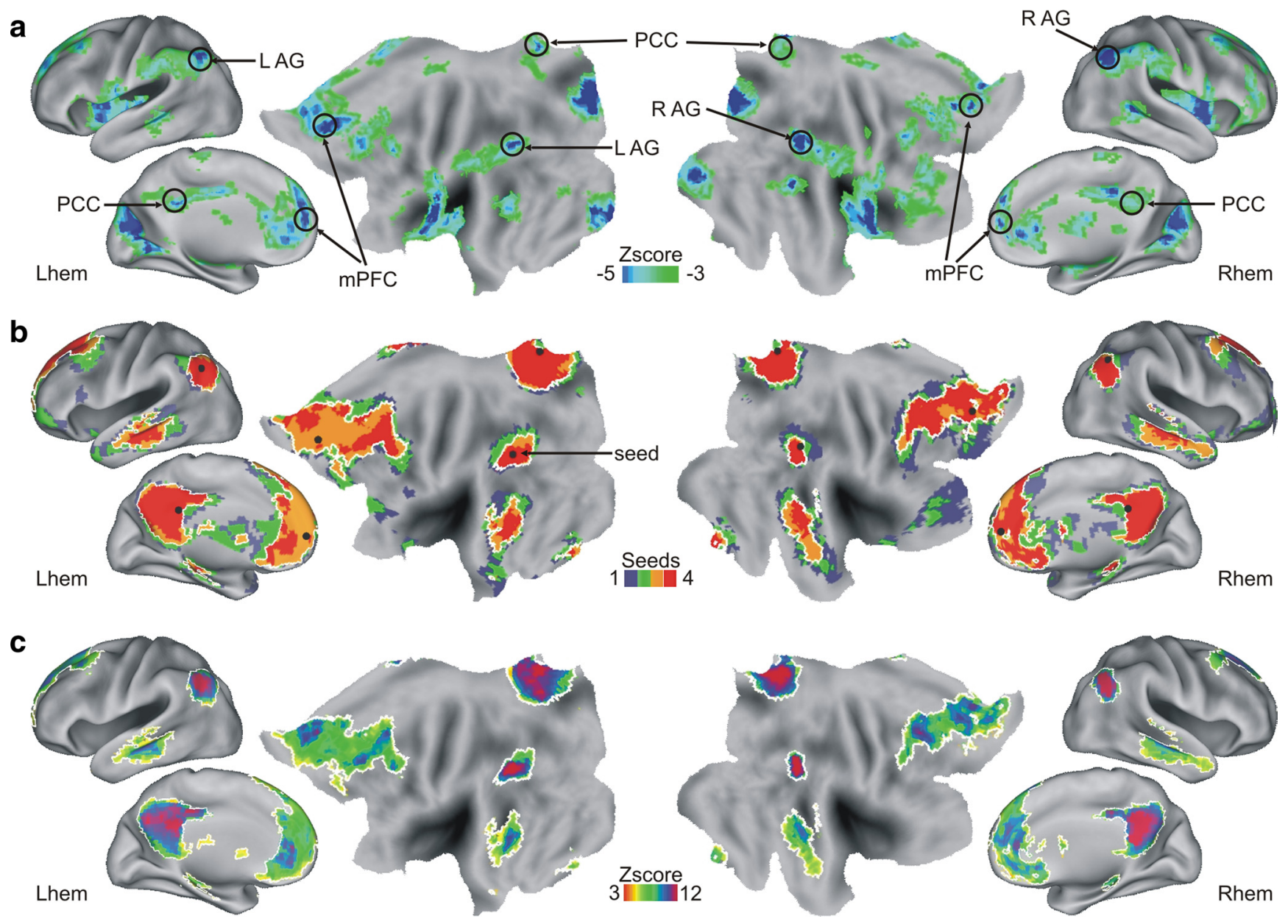

Figure 2. Definition of the DMN. $\boldsymbol{a}$, Multiple-comparison corrected group z-map showing search-related BOLD deactivations (light to dark blue colors) with respect to the baseline in the perceptual search task. The search parameter was obtained using Search-Hit-HC trials (process GLM) (see Materials and Methods). The voxelwise map is superimposed over the lateral inflated, the medial inflated, and the flat representation of both hemisphere of the PALS Atlas [Caret 5.5 software (Van Essen, 2005)]. The dark circles represent the peaks of deactivations that were used as seeds for the functional connectivity analysis. Seeds in PCC and mPFC are presented in both hemisphere since they were close $(1 \mathrm{~mm})$ to the midline. $\boldsymbol{b}$, Frequency map that represents voxels showing functional connectivity with the seed regions obtained from the perceptual task. For each voxel, the color represents significant resting-state positive correlations with one (blue), two (green), three (orange), or four (red) seeds. The DMN was defined as those voxels showing functional connectivity with at least three of four seeds (regions surrounded by white borders). c, Group $z$-map showing how connectivity varies within the regions that are defined as being part of the DMN. The map represents the mean zscore of the rs-fcMRI maps obtained using the four seeds. The resulting map was masked by the border of the DMN (white).

allowing the isolation of the search process. The graph shows the number of trials (all trials, correct trials, and incorrect trials) in which the reaction time to make the memory judgment, measured from the start of the search phase (i.e., from the offset of the sentence), fell within a certain interval. Because very few responses occurred in the $12-15 \mathrm{~s}$ interval and performance at two time points was at chance, trials in this interval were excluded from the subsequent fMRI analysis. The remaining trials were divided into three bins (early, 0-4 s; middle, $4-8 \mathrm{~s}$; late, $8-12 \mathrm{~s}$ after sentence offset) for time course averaging in the fMRI analysis. A significant confidence effect was observed (supplemental Fig. $2 b$, available at www.jneurosci.org as supplemental material): accuracy was $0.81 \pm 0.2$ for high- and $0.63 \pm 0.2$ for lowconfidence trials $\left(t_{(18)}=7.40 ; p<0.001\right.$, two-tailed paired $t$ test $)$. There was also a progressive decrease in accuracy as retrieval time took longer $(0.82 \pm 0.01,0.73 \pm 0.02$, and $0.67 \pm 0.02$, respectively) $\left(F_{(2,34)}=85.25, p<0.001\right.$, one-way ANOVA); however, performance was mostly above chance also in the late bin $(p<$ 0.001 , one-sample $t$ test).

\section{fMRI}

Identification of the default mode network

The DMN was identified using a two-step approach. First, since the DMN is known to be deactivated during active, goal-directed analyses of environmental stimuli (Shulman et al., 1997; Raichle et al., 2001), we examined search-related BOLD activity in the perceptual task, in which visually presented sentences instructed participants to search for and detect visual targets in $12 \mathrm{~s}$ video clips. By manipulating the time at which the target appeared, we separated brain activity associated specifically with searching for the visual target from activity because of the physical display and target detection. Four peaks of search-related deactivations corresponding to the major nodes of the DMN were identified: the $\operatorname{mPFC}(x=+1, y=+49, z=+11)$, the PCC $(x=-1, y=-45$, $z=+28)$, and the left $(x=-44, y=-63, z=+34)$ and right AG $(x=+44, y=-62, z=+40)$ (Fig. 2a). Other peaks of deactivations were identified in the map of search-related activity, although they extended into regions that are not classically associated with the DMN (e.g., peripheral early visual cortex, 
posterior insula). The presence of nontraditional regions of BOLD signal deactivation may have depended on the specific characteristics of the current perceptual search task, a source of variation that is minimized in metaanalyses that consider common deactivated regions across multiple tasks (Shulman et al., 1997). For this reason, we combined the deactivation foci approach with a rs-fcMRI approach to identify the full network in accordance with the current literature (Buckner et al., 2008).

The coordinates of the four peaks of deactivation were used as seeds in a connectivity analysis that involved an independent set of rs-fMRI runs collected on the same subjects. Figure $2 b$ shows the conjunction map representing voxels showing positive correlation with one up to four seeds. The DMN was identified as those voxels showing correlation with at least three of four seeds (areas enclosed in the white borders). The resulting DMN is highly consistent with previous definitions (Shulman et al., 1997; Mazoyer et al., 2001; Buckner et al., 2008) and comprises midline [ventral and dorsal mPFC; PCC, extending toward the precuneus (PreCu) and the retrosplenial cortex], and lateral structures (AG, superior frontal sulcus, lateral temporal cortex, and hippocampus, bilaterally). Figure $2 c$, which represents the average of the connectivity maps obtained using each seed, illustrates how connectivity varies within the regions that are defined as being part of the DMN.

Functional heterogeneity of parietal regions located inside and outside the DMN during episodic memory retrieval

Next, we investigated the role of parietal regions inside and outside the DMN during the episodic memory task, in which subjects were allowed up to $15 \mathrm{~s}$ to judge the accuracy of sentences describing specific details about one of two movies that had been viewed on a previous day. The large intertrial variability of retrieval time was exploited to isolate memory search-related activity from the activity related to the execution of the motor response, since these processes can deactivate the DMN. If posterior nodes of the DMN directly support episodic retrieval whereas more dorsal parietal regions are more involved in postretrieval processes, then these regions should show different time courses, with retrieval-related regions showing peak activity before decision-related regions.

Figure $3 a$ shows the positive memory search-related activations that were observed in posterior regions located inside and outside the DMN, which is indicated by white outlines. Within the DMN, the strongest memory search related activations were observed in angular gyrus and posterior cingulate/precuneus (intra-DMN, AG and PCC-PreCu). Outside the default network, parietal activity (Fig. 3a) was observed in left anterior intraparietal and postcentral sulcus (extra-DMN, aIPS and PoCS), which were located more dorsally and anteriorly with respect to the AG region of the $\mathrm{DMN}$.

Figure $3 b$ (top row) shows the BOLD time courses in intraDMN parietal regions for trials involving different search durations, starting from the onset of the sentence (i.e., from the start of the trial). Activity quickly reached peak activation levels $\sim 8-10$ s from cue sentence onset (highlighted by the gray transparent bar) for each of the three search intervals, but peak magnitude increased with retrieval duration, consistent with a functional role in memory search. Therefore, the BOLD signal showed an amplitude increase but no "progressive" shift in peak onset [peak onset shifting later in time (i.e., to the right on the graphs)] from early to late trials. A similar pattern of activity was observed in a left pParaHC, which showed significant memory search-related activity in accordance with several neuroanatomical models (Davachi, 2006; Eichenbaum et al., 2007). This pattern

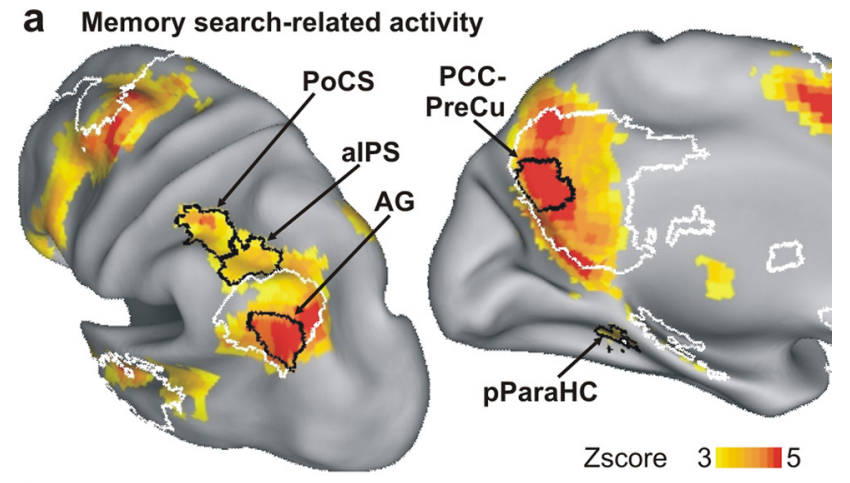

b Timecourses of Memory Correct Trials
AG

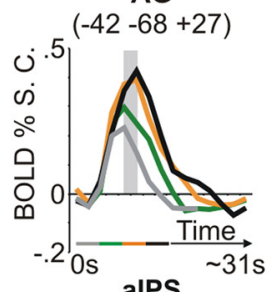

alPS

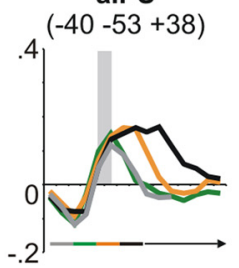

—Early —Middle
PCC-PreCu

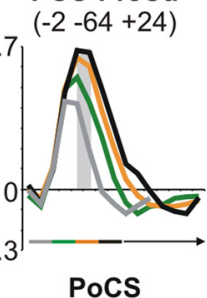

PoCs

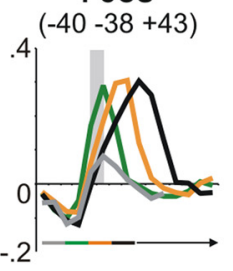

- Late - Catch

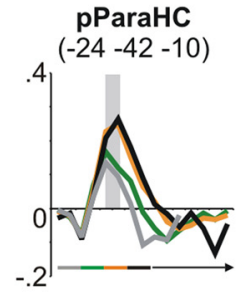

C. Peak analysis

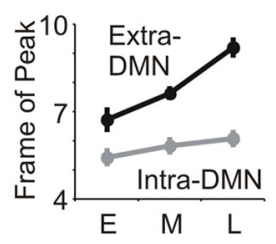

Retrieval Interval
Figure 3. Profile of BOLD activity of intra- and extra-DMN regions during the memory task. $\boldsymbol{a}$, Inflated representation of the left hemisphere showing regions of the lateral and medial parietal lobe centered on the peaks of positive memory search-related activity (process GLM). Regions (defined by black borders) are located either inside (intra-DMN: AG, PCC-PreCu) or outside (extra-DMN: aIPS, PoCS) the DMN (white borders) and are superimposed over the voxelwise map of positive search-related activity. A region of the medial temporal lobe with significant memory search-related activity (pParaHC) is also highlighted. $\boldsymbol{b}$, Time courses of BOLD activity, aligned to the onset of the cue sentence (frame-by-frame, between-trial GLM), extracted from the five regions illustrated in $\boldsymbol{a}$. Intra- and extra-DMN regions are located on the top and bottom rows, respectively. Time courses were grouped according to the retrieval time interval and refer to catch trials (gray), and early ( $1-4 \mathrm{~s}$; green), middle ( $4-8 \mathrm{~s}$; orange), and late $(8-12 \mathrm{~s}$; black) correct trials. The gray transparent vertical bar in each graph highlights the fifth and sixth frame (corresponding to $\sim 8-10 \mathrm{~s}$ after cue sentence onset) for comparison purpose. The horizontal line under the graphs illustrates the temporal duration of the corresponding retrieval intervals. c, The graph illustrates the relationship between peak latency of BOLD activity (frame of peak) and retrieval interval in extra-DMN regions (aIPS and PoCS; black line) compared with intra-DMN regions (AG and PCC-PreCu; gray line). Extra-DMN regions showed a greater increase in peak latency as subjects took more time to judge the correctness of cue sentences, indicating a more sustained involvement during retrieval.

of activity suggests an involvement of posterior DMN regions, in association with medial temporal cortex, in the retrieval of information triggered by the cue sentence.

In contrast, extra-DMN regions (Fig. $3 b$, bottom row) exhibited an initial decrease of the BOLD signal after cue sentence onset, followed by a sustained activation characterized by a progressive shift in peak onset from early to late trials. This pattern suggests a delayed but more sustained involvement of these regions in the memory task, consistent with a role in the manipulation of retrieved information in working memory or in memory-based decision making. The reliability of this difference was tested in a three-way ANOVA on a weighted measure of peak latency (see Materials and Methods), with DMN (intra-, extra-), 
region (two levels), and interval (early, middle, late) as factors. This analysis revealed a highly significant interaction between DMN and interval $\left(F_{(2,34)}=14.73 ; p<0.0001\right)$ and no interaction with region (Fig. $3 c$ ), confirming a reliable difference in peak latency across sets of regions.

Activity in both intra-DMN and extra-DMN regions was present during the sentence-reading phase of the trial, as illustrated by the time courses for catch trials (gray lines in the time courses), in which the trial ended after the offset of the $4 \mathrm{~s}$ sentence cue (Fig. 3b). Because these regions were either not activated (PCC-PreCu: $t_{(18)}=-0.91, p=$ NS; PoCS: $t_{(18)}=-1.33$, $p=\mathrm{NS}$; one-sample $t$ test of the perceptual sentence reading parameter against the baseline), with a trend in the means for deactivation, or even significantly deactivated (AG: $t_{(18)}=-4.35$, $p<0.001$; aIPS: $\left.t_{(18)}=-5.14, p<0.0001\right)$ by sentence reading in the perceptual task, this result suggests that memory retrieval and evaluation probably started during the sentence phase of the memory task.

\section{Sequence of BOLD responses from memory search to} motor execution

The delayed peak of activity observed in extra-DMN, compared with intra-DMN regions (Fig. $3 c$ ), raised the possibility that their activity extended to the motor response phase of the task, which was assessed with the detection parameter obtained using the process GLM (see Materials and Methods). The voxelwise map in Figure $4 a$ shows the distribution of positive detection BOLD activity during correct memory trials. Detection activity was clearly observed in both extra-DMN regions (PoCS: $t_{(18)}=9.55, p<$ 0.0001; aIPS: $t_{(18)}=6.02, p<0.0001$, one-sample $t$ test) and in somato-motor cortex (SMC) [( $x=-32, y=-29, z=+55)$, indicated in the figure], but not in intra-DMN (AG: $t_{(18)}=$ $-0.20, p=0.84$; PCC-PreCu: $t_{(18)}=0.83, p=0.42$ ). However, extra-DMN regions also differed from the SMC region, as the latter was not activated by the search phase of the task (Fig. 4a). $t$ tests comparing the memory search parameters for aIPS and PoCS with SMC yielded significant differences for both pairs of regions ( aIPS vs $S M C, t_{(18)}=5.17, p<0.0001$; PoCS vs $S M C$, $t_{(18)}=$ $4.84, p<0.0001$; paired-sample $t$ tests). Therefore, extra-DMN regions were activated during memory search before motor execution but this activation extended into the final decision/response phase of the task.

The progressive sequencing over time of activation from intra- to extra-DMN regions to somatomotor cortex is shown in Figure $4 b$, which displays time courses from these regions in a single graph for each retrieval interval. In the early interval (left graph), activity in pParaHC (gray line), intra-DMN (red), extraDMN (blue), and SMC (black) starts from baseline and reaches a peak at closely spaced times. However, as the duration of the memory search phase increases, the activity of these regions becomes more temporally separated. This segregation is maximal in the late interval (right graph), which clearly shows the temporal progression of activity from parietal intra-DMN and the pParaHC regions to parietal extra-DMN regions and finally to the SMC, which had the latest onset.

We conducted an analysis to show that the time courses of the extra-DMN regions differed from the time course in SMC. A two-way ANOVA on the data from the late interval, with region (two levels) and time (15 MR frames) as factors, revealed significant interactions of region by time for the comparison of SMC and aIPS $\left(F_{(14,238)}=5.15 ; p<0.0001\right)$ and SMC and PoCS $\left(F_{(14,238)}=10.33 ; p<0.0001\right)$. These results confirm those from the earlier analysis showing that the memory search parameter in

\section{a Memory Detection-related activity (Correct Trials)}

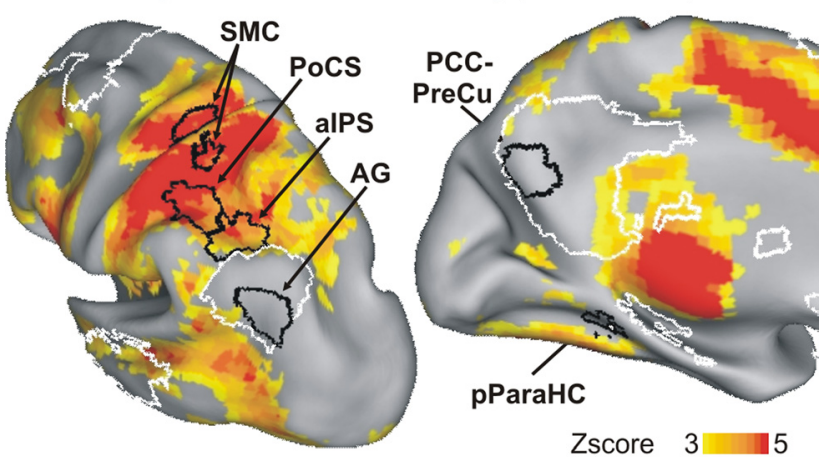

b Timecourses of Memory Correct Trials

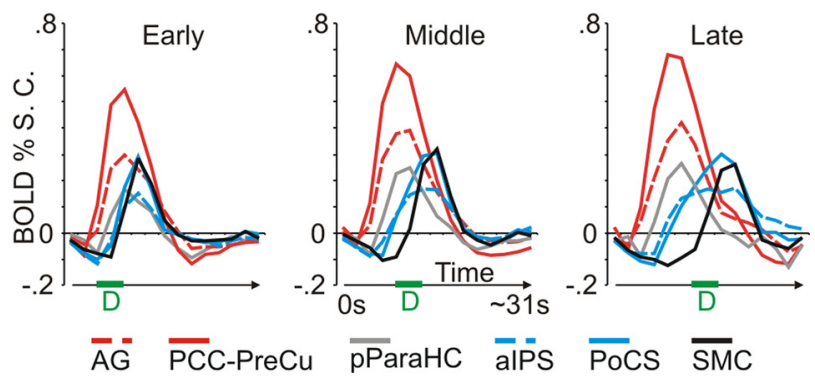

Figure 4. Sequence of BOLD responses from memory search to motor execution. $a$, Multiplecomparison corrected group z-map showing detection-related BOLD activations (red to yellow colors) with respect to the baseline in the memory search task. The detection parameter was obtained using all correct trials (process GLM) (see Materials and Methods). The DMN (white) and the parietal ROls (black) are shown. An additional region [motor cortex (MC)] was created on the peak of detection-related response corresponding to the hand region of the motor cortex. $\boldsymbol{b}$, Time courses of BOLD activity arranged so that different graphs represent the activity of different regions in each retrieval interval. The progressive involvement of different regions during the memory task is particularly evident in the late interval (right graph). Intra-DMN regions are presented in red (AG, PCC-PreCu), extra-DMN in light blue, PParaHC in gray, and motor cortex in black color. The green line and the letter " $\mathrm{D}$ " in each graph indicate the temporal interval in which detections occurred.

SMC was significantly smaller than the memory search parameters for aIPS and PoCS.

Overall, these results suggest a flow of activity from intra- to extra-DMN regions to somatomotor cortex during the performance of the episodic memory task.

Functional connectivity of intra- and extra-DMN parietal regions We examined whether the functional distinctions between intraDMN and extra-DMN regions were corroborated by different patterns of functional connectivity in the resting state. The coordinates of intra- and extra-DMN regions and of the pParaHC region, derived from the map of memory search-related activity, were used as seeds to create rs-fcMRI voxelwise maps. Figure 5, $a$ and $b$, illustrates the rs-fcMRI maps obtained using the AG and the PoCS regions as seeds, respectively. As expected, the pattern of functional connectivity using the left AG seed matched almost perfectly the topography of the DMN, showing positive correlation with the PCC, the MPFC, and medial and lateral aspects of the temporal cortex. In contrast, the connectivity map of the PoCS seed was noticeably different, showing positive correlation only with voxels that were outside the DMN and negative correlation with all the regions of the DMN. Furthermore, the rsfcMRI map obtained using the pParaHC region as a seed (Fig. $5 c$ ) showed positive correlation only with intra-, but not extra-DMN regions. Notably, the pParaHC region also showed positive correlation with the ventral aspect of the mPFC. Figure $5 d$ illustrates 
a AG

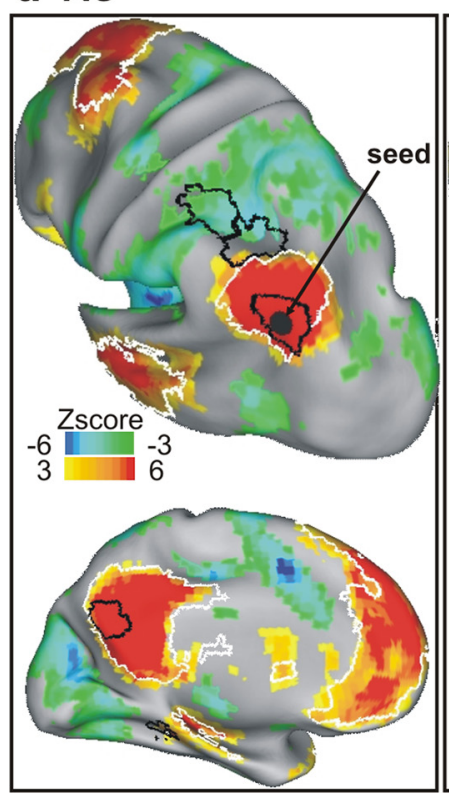

b Pocs

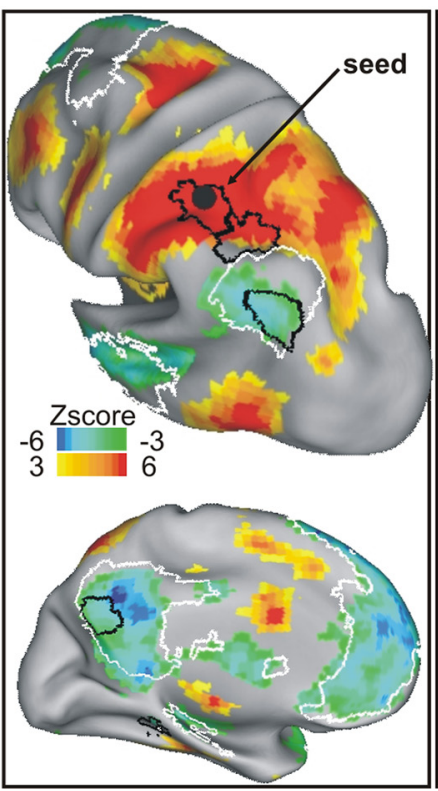

c pParaHC

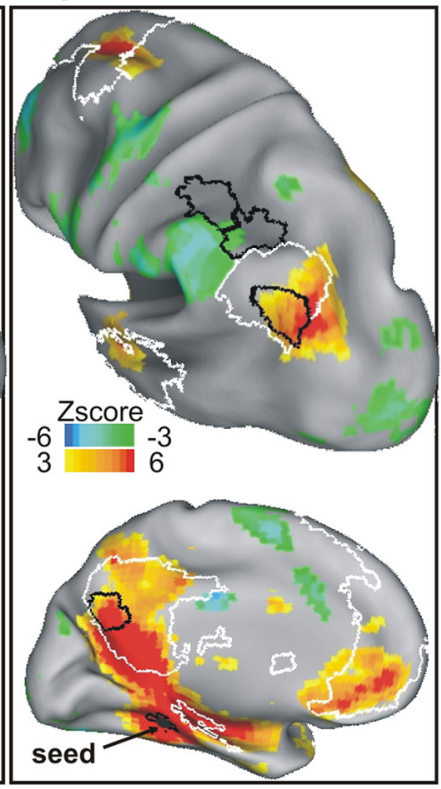

d Cross-correlation matrix
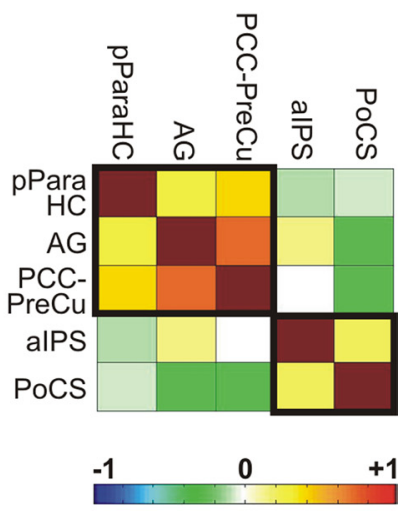

correlation (R)

Figure 5. Functional connectivity of intra- and extra-DMN parietal regions. $\boldsymbol{a}$, Inflated representation of the left hemisphere showing the voxelwise rs-fcMRI statistical maps obtained in the same group of subjects using the left AG as seed. The group voxelwise z-map is shown for a one-sample, random-effects $t$ test, corrected for multiple comparisons, of whether the Fisher $z$-transformed correlation values were different from zero. The black sphere represents the location of the $6 \mathrm{~mm}$ seed. $\boldsymbol{b}, \boldsymbol{c}$, Voxelwise rs-fcMRI statistical maps using PoCS and pParaHC gyrus as seeds, respectively. $\boldsymbol{d}$, Cross-correlation matrix illustrating the correlation between pairs of regions among the sets of intra- and extra-DMN regions and the pParaHC region. The degree of correlation is color-coded so that green to blue colors represent increasing negative correlation and yellow to red colors represent increasing positive correlation.

the cross-correlation matrix showing the regional correlation across the sets of intra- and extra-DMN regions and the pParaHC region. The regional analysis confirmed that PCC-PreCu was uncorrelated with aIPS $\left(r=-0.03 ; t_{(18)}=-0.44 ; p=0.67\right.$, one-sample $t$ test), negatively correlated with PoCS $(r=-0.28$; $\left.t_{(18)}=-7.90 ; p<0.0001\right)$, and positively correlated with AG $(r=$ $\left.0.69 ; t_{(18)}=19.26 ; p<0.0001\right)$ and pParaHC $\left(r=0.38 ; t_{(18)}=\right.$ 8.97; $p<0.0001)$. Similarly, AG was negatively correlated with PoCS $\left(r=-0.26 ; t_{(18)}=-7.42 ; p<0.0001\right)$ and positively correlated with pParaHC $\left(r=0.28 ; t_{(18)}=6.63 ; p<0.0001\right)$. The analysis revealed that AG was slightly correlated with aIPS $(r=0.15$; $\left.t_{(18)}=2.27 ; p<0.05\right)$, but, given the spatial proximity of the regions, this relationship may primarily reflect local correlation. Overall, the pattern of resting-state functional connectivity further indicated a substantial dissociation between extra- and intra-DMN regions, supporting their functional task-based dissociation.

\section{Dissociation between anterior and posterior nodes of the DMN during episodic memory search}

We next examined whether the DMN functions homogenously during episodic memory retrieval by comparing the involvement of anterior and posterior nodes of the DMN during the memory search task. Figure $6 a$ illustrates the cortical distribution of memory search-related BOLD activations (red to yellow colors) and deactivations (green to blue colors) in cortical regions that fell within the borders of the DMN, delimitated by white borders. Figure $6 b$ shows the BOLD time courses for trials involving different search durations, starting from the onset of the sentence (i.e., the start of the trial).

A clear dissociation was observed between the activity of anterior and posterior nodes of the DMN. As shown above, robust memory search-related activation over the baseline was observed in several posterior nodes of the DMN (AG and PCC-PreCu) in both hemispheres. In contrast, regions around the anterior node of the DMN (mPFC) exhibited a robust search-related deactiva- tion that varied with the duration of memory retrieval. This dissociation between anterior and posterior nodes of the DMN, although evident from the time courses of the entire trial shown in Figure $6 b$, was observed for multiple phases of the memory task. It was present during the sentence-reading phase, as illustrated by the time courses for catch trials (gray lines in the time courses), in which the trial ended after the offset of the $4 \mathrm{~s}$ sentence cue (Fig. 6b). It was also observed during the pure memory retrieval phase of the task that followed the sentence cue, illustrated by the time courses that specifically isolated the memory retrieval phase (see Materials and Methods), as shown in supplemental Figure 3 (available at www.jneurosci.org as supplemental material).

To test the robustness of the present results, we replicated the analyses using a DMN definition based on a pure deactivation criterion (i.e., foci that were deactivated during the perceptual task) (Fig. 2a), rather using a definition based on resting-state functional connectivity analyses of these deactivated foci (Fig. $2 b$ ). The same dissociation between MPFC and AG/PCC was observed when memory activity was extracted from the regions that were used as seeds for the functional connectivity analysis (supplemental Fig. $4 a$, available at www.jneurosci.org as supplemental material). In particular, the PCC and the left and right AG seeds all showed a positive, parametric modulation of the BOLD signal during the memory task (supplemental Fig. $4 c$, available at www.jneurosci.org as supplemental material) and a significant positive modulation of the memory search parameter (SearchCorr-HC) (supplemental Fig. $4 d$, available at www.jneurosci.org as supplemental material), whereas the opposite pattern was observed in the mPFC seed.

However, although significant activity in the episodic memory task was observed for regions of PCC-PreCu that fell within the borders of the DMN as defined using either a composite perceptual deactivation/rs-fcMRI criterion or a pure perceptual 


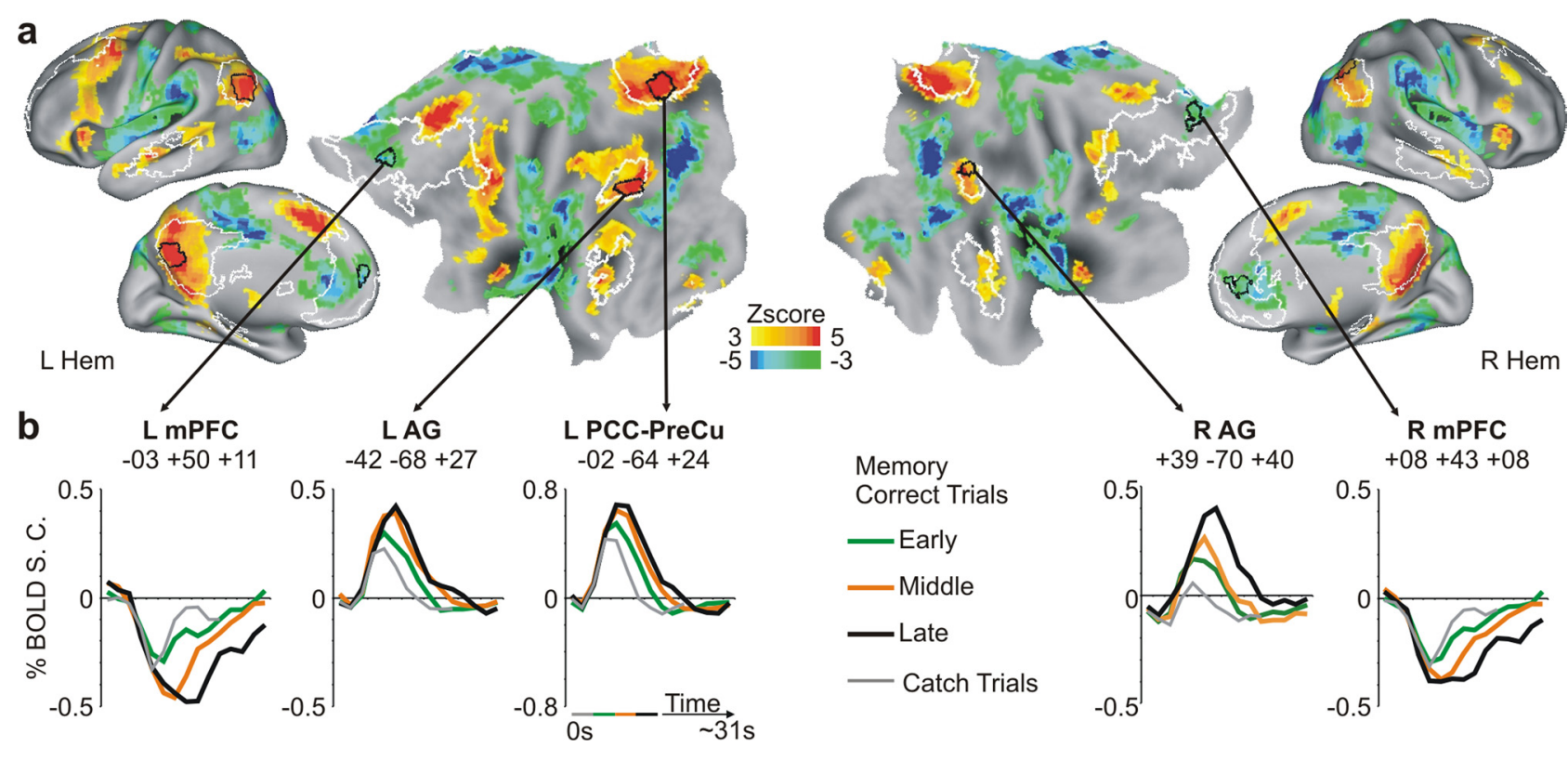

Figure 6. The relationship between the DMN and memory search-related activity. $\boldsymbol{a}$, Whole-brain, multiple-comparison corrected group z-map showing search-related BOLD activations (red to yellow colors) and deactivations (light to dark blue colors) with respect to the baseline in the memory search task. The search parameter was obtained using Search-Corr-HC trials (process GLM) (see Materials and Methods). The borders of the DMN (in white) are superimposed over the activation map to reveal regions of overlap. $\boldsymbol{b}$, Time courses of BOLD activity, aligned to the onset of the cue sentence (frame-by-frame, between-trial GLM), extracted from five regions showing significant positive and negative memory search-related responses that were located inside the DMN (defined by black borders on the flat map). Time courses were grouped according to the retrieval time interval and refer to catch trials (gray), and early (1-4s; green), middle (4-8s; orange), and late ( $8-12$ s; black) correct trials. The horizontal line under the third graph illustrates the temporal duration of the corresponding retrieval intervals.

deactivation criterion, the peak memory-related activity (PCCPreCu; $x=-2, y=-64, z=+24)$ was nonetheless centered in a more posterior location than the peak search-related deactivation during the perceptual task (PCC; $x=-1, y=-45, z=+28$ ) (compare Figs. 2a, 3a) (for similar results, see also Buckner et al., 1996). The vector distance between these peaks was $19.4 \mathrm{~mm}$, whereas the distance between the peaks of perceptual deactivation and memory activation in left and right AG was 8.8 and 9.4 $\mathrm{mm}$, respectively. There were multiple peak deactivations in $\mathrm{mPFC}$ during the perceptual task, as shown in supplemental Figure $4 a$ (available at www.jneurosci.org as supplemental material), but the peak at $+1,+49,+11$ that was used in the rs-fcMRI analysis was only $4.1 \mathrm{~mm}$ from a peak deactivation in the memory task, as shown in supplemental Figure $5 a$ (available at www. jneurosci.org as supplemental material). Correspondingly, based on the ROIs defined from peak memory-related activity (supplemental Fig. $5 a$, available at www.jneurosci.org as supplemental material), PCC-PreCu exhibited only a trend for a search-related deactivation in the perceptual task (supplemental Fig. $5 d$, available at www.jneurosci.org as supplemental material) $\left[t_{(18)}=\right.$ $-0.09 ; p=0.08$; one-sample $t$ test between the search parameter (Search-Hit-HC) and the baseline], whereas significant deactivations were still observed in AG and mPFC (supplemental Fig. $5 d$, available at www.jneurosci.org as supplemental material).

Therefore, for AG and mPFC, peak activations (AG) or deactivations ( $\mathrm{mPFC}$ ) during the episodic memory task fell within the borders of the DMN regardless of how those borders were defined (i.e., using a composite perceptual deactivation/rs-fcMRI criterion or a peak perceptual deactivation criterion). In contrast, the peak activation of the PCC-PreCu during the episodic memory task fell within the borders of the DMN using a composite perceptual deactivation/rs-fcMRI criterion but not a peak perceptual deactivation criterion, since the activation was located more posteriorly. Nevertheless, it is important to remember that all PCC-PreCu ROIs within the DMN were significantly activated by the episodic memory task, including the ROI that was defined by a peak perceptual task deactivation criterion (supplemental Fig. $4 a$, available at www.jneurosci.org as supplemental material). Therefore, PCC-PreCu showed a pattern of episodic memory task activity that was completely different from that shown in mPFC (i.e., significant activity that was opposite in sign).

\section{The BOLD-behavior relationship supports the presence of} a dissociation

We investigated the relationship between memory search activity in anterior and posterior DMN regions and behavioral performance, by separating the BOLD signal on trials in which a correct memory judgment was made with high or low confidence. The effect of confidence on the BOLD signal exceeded the Bonferroni-corrected significance threshold $(p<0.0025)$ within the left lateral parietal DMN ROI, extending toward the occipital cortex [AG $(x=-33, y=-76, z=+36), t_{(17)}=$ 3.65, $p=0.0020$; two-tailed paired $t$ test] (Fig. 7, first graph from the left), supporting the behavioral significance of memory search-related activity observed in DMN regions. Two other regions located within the borders of the left lateral and medial parietal DMN nodes showed a milder confidence effect [AG $(x=-46, y=-68, z=+27), t_{(17)}=2.56, p<0.05$; PCC $(x=-10, y=-49, z=+36), t_{(17)}=2.40, p<0.05$ ] (Fig. 7, second graph), which, however, did not exceed the Bonferronicorrected statistical threshold.

Previous results have indicated that the magnitude of perceptual search-related deactivations are related to task performance (Shulman et al., 2007), supporting a functional role during task execution (e.g., filtering of potentially distracting information). However, in the present study, deactivated regions in $\mathrm{mPFC}$ were not affected by retrieval confidence [ventral (v)mPFC $(x=0, y=$ $+47, z=+08), t_{(17)}=-0.64, p=0.53$; dorsal $(\mathrm{d}) \operatorname{mPFC}(x=$ $-03, y=+50, z=+28), t_{(17)}=0.001, p=1.00$ ] (Fig. 7, third and fourth graphs, respectively), indicating that BOLD deactiva- 


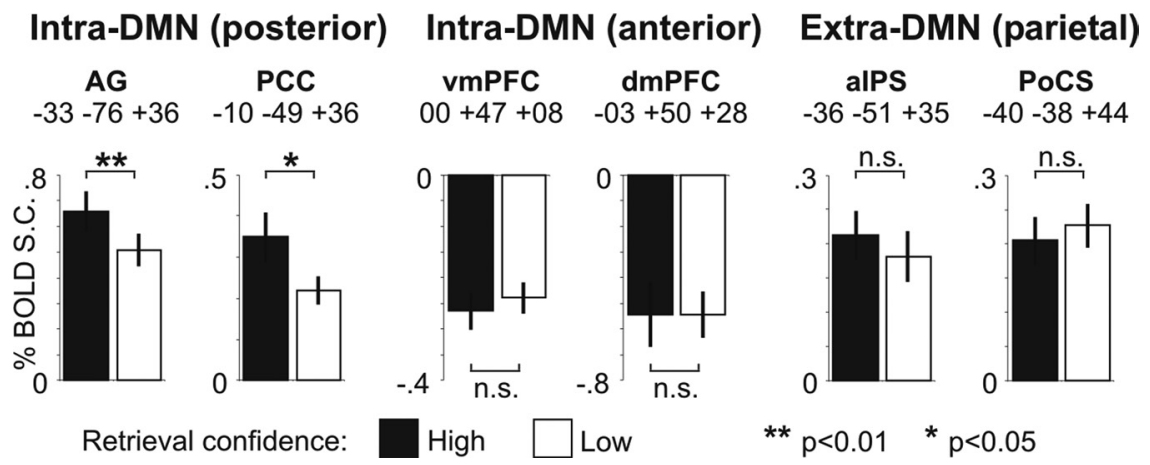

Figure 7. BOLD-behavior relationship. BOLD percentage signal change for the search parameter (process GLM) corresponding to high (gray bar) and low (white bar) confidence correct trials in the memory task. These BOLD magnitudes were extracted from two posterior intra-DMN regions (AG, PCC; first and second graphs, respectively), two anterior intra-DMN regions (vmPFC, dmPFC; third and fourth graphs, respectively), and two parietal extra-DMN regions (alPS, PoCS; fifth and sixth graphs, respectively). Error bars indicate SEM. The asterisks indicate the significance of the paired $t$ test between high-and low-confidence correct search parameters.

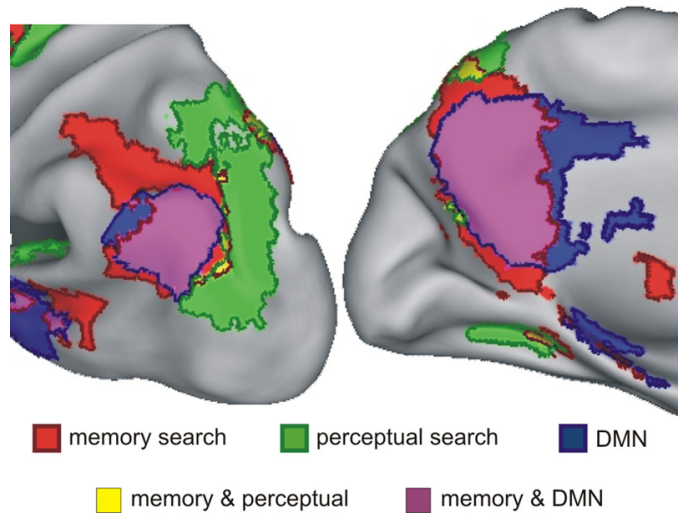

Figure 8. Functional organization of the left posterior parietal cortex. Inflated representation of the left hemisphere (lateral and medial views) showing parietal regions positively activated by memory (red color delimitated by dark red borders) and perceptual (green, delimitated by dark green borders) search as well as regions belonging to the DMN (blue, delimitated by dark blue borders), as defined by functional connectivity. Regions of overlap between memory search activity and the DMN are illustrated in purple color, whereas regions of overlap between memory and perceptual search are shown in yellow.

tions in these regions were not related to behavioral outcome. Overall, the above results indicate that during episodic memory retrieval anterior nodes of the default system (mPFC) are strongly dissociated from posterior nodes, particularly the AG.

Finally, no significant difference between memory search followed by a high- or low-confidence judgment was observed in parietal extra-DMN regions [aIPS $(x=-36, y=-51, z=+35)$, $t_{(17)}=0.86, p=0.40$; PoCS $(x=-40, y=-38, z=+44), t_{(17)}=$ $-0.59, p=0.56$ ] (Fig. 7, fifth and sixth graphs, respectively), providing additional evidence for the functional dissociation of intraand extra-DMN regions.

\section{Discussion}

The results of the present study demonstrate two general conclusions concerning the role of the DMN during episodic memory retrieval. First, parietal regions of the DMN are activated in conjunction with extra-DMN regions during episodic memory retrieval, but perform different functions. The early peak of taskevoked activity in intra-DMN and parahippocampal regions and the close coupling of these regions in rs-fcMRI analyses, both suggest a role of parietal DMN nodes in retrieval of information in association with medial temporal systems. Conversely, the later sustained time courses of activity in extra-DMN nodes, which extended into the period in which a final judgment/response was made, is more consistent with a role in organizing retrieved material in working memory, accumulating evidence that points toward a particular decision, and making a final decision and response. Second, the DMN shows functional heterogeneity during episodic memory retrieval, with a clear dissociation between posterior DMN nodes (particularly the AG), which were activated by episodic memory retrieval in a functionally significant manner, and an anterior DMN node, $\mathrm{mPFC}$, which was deactivated by episodic memory retrieval and showed no behavioral significance.
Three functionally dissociable sets of parietal regions during episodic memory retrieval

Using a novel experimental paradigm (Sestieri et al., 2010) that parametrically varied the duration of episodic memory processing, we demonstrated differences in the task-evoked time courses of memory-related activity that carried clear functional implications. We separately defined the DMN using independent datasets from the same subjects (i.e., runs from the perceptual task and rs-fMRI runs), allowing us to relate these functional characteristics to regions inside and outside the DMN.

Memory-related regions located within the posterior DMN nodes showed a time course characterized by a rapid increase of activity in response to a cue requiring the retrieval and evaluation of information in episodic memory. BOLD activity peaked at approximately the same time for all memory search/retrieval intervals, but exhibited larger amplitudes for later intervals. The scaling of the response amplitude with interval may have reflected the greater amount of information (Vilberg and Rugg, 2007 ) that needed to be retrieved to answer the sentence query on trials in which the final decision and response was rendered late. AG and PCC were functionally connected in the resting state with a medial temporal region that showed a similar time course of memory-related activity. The early peak response after the sentence cue and the preferential connectivity with medial temporal structures support the idea that these regions were directly involved in the retrieval process. AG and PCC-PreCu may act as an "episodic buffer" that represents an interface between stored memories and an executive system (Baddeley, 2000; Vilberg and Rugg, 2008), or as a mechanism for orienting the focus of attention to mnemonic representations (Wagner et al., 2005).

In contrast, posterior regions located outside the DMN, in aIPS and PoCS, showed a progressive shift in the time of peak activity with increasing interval (peak onset shifting later in time from early to late trials) and an absence of resting connectivity with medial temporal structures. This pattern of results suggests a greater involvement of these extra-DMN regions in postretrieval processes such as the on-line manipulation of retrieved information in working memory to meet a behavioral goal (e.g., answering the sentence query) or the accumulation of evidence toward a memory-based decision (Wagner et al., 2005; Donaldson et al., 2010). The sustained activity of aIPS and PoCS was observed up to the motor execution phase of the memory task. This late activation might 
indicate involvement in the motor plan associated with a given memory-based decision in addition to the processes discussed above. We emphasize that the complex set of processes that we have labeled, for convenience, as memory search or memory retrieval, included more than retrieval or search, and was not organized serially but perhaps in cascade or as continuous flow: the retrieval of episodic information guided by the behavioral goal, the organization and evaluation of the retrieved information, and the accumulation of evidence pointing to a particular response (Moscovitch and Winocur, 1995; Badre and Wagner, 2007; Mecklinger, 2010). Others have referred to this type of retrieval as cued recollection (Mendelsohn et al., 2010).

Sestieri et al. (2010) have shown using the current dataset that the two sets of memory-related parietal regions (intraand extra-DMN regions) (Fig. 8, purple and red colors) were strongly dissociated from a third adjacent but nonoverlapping set of regions that included more posterior sections of IPS and SPL (Fig. 8, in green). These latter regions were activated by the perceptual search task used here to define the DMN, whereas some were deactivated by the episodic memory task, indicating a competitive relationship between parietal regions involved in perception and episodic memory. As shown in Figure 8, these three sets of functionally and anatomically distinct regions tiled large parts of left parietal cortex, similar to a recent proposal (Nelson et al., 2010).

\section{Functional heterogeneity of the DMN during episodic memory retrieval}

The present results also showed a clear functional dissociation of anterior and posterior DMN nodes on the basis of task-induced activity, in contrast with the homogeneity of task-induced deactivations during goal-directed analyses of environmental stimuli (Shulman et al., 1997; Mazoyer et al., 2001).

A posterior node of the $\mathrm{DMN}$, the AG, was unambiguously activated over the baseline during the memory task and was modulated in a predictable manner by retrieval time. Furthermore, it was modulated by retrieval confidence, indicating a relationship with task performance. Regions in the PCC-precuneus showed similar properties, but the association of the PCC-precuneus node of the DMN with episodic memory retrieval was more ambiguous since the peak memory retrieval activity was not aligned with the peak deactivation during the perceptual task, which occurred almost $2 \mathrm{~cm}$ more posteriorly. Therefore, although a large section of PCC-precuneus cortex showed similar patterns of resting-state functional connectivity, it nevertheless showed some task-related heterogeneity, consistent with a previous observation (Buckner et al., 1996).

Positive activation of putative DMN regions above a resting baseline has been previously reported during the execution of internally oriented tasks (Iacoboni et al., 2004; Ochsner et al., 2004; Goldberg et al., 2006), but the lack of an independent definition of the DMN within the same study has precluded a conclusive demonstration of this phenomenon. The current results, in conjunction with recent proposals (Buckner et al., 2008; Goldberg et al., 2008), indicate that the DMN can be activated by goal-oriented tasks that emphasize internally directed processes such as episodic memory retrieval.

The activations observed in posterior DMN nodes contrasted with the strong deactivation of regions within the anterior mPFC node of the DMN. mPFC was parametrically deactivated during memory retrieval and the magnitude of the deactivation was un- related to task performance, indicating that $\mathrm{mPFC}$ activity during the memory task was not functionally significant.

Previous reviews of the neural correlates of episodic memory have focused on the role of lateral rather than medial regions of the prefrontal cortex (Buckner and Wheeler, 2001; Rugg et al., 2002). mPFC has not been consistently associated with retrieval success, although mPFC modulations have been reported in studies investigating recollection versus familiarity judgments (Henson et al., 1999; Kahn et al., 2004; Yonelinas et al., 2005). Recent evidence suggests that mPFC is selectively involved in episodic memory tasks that explicitly require retrieval of autobiographical information (Cabeza et al., 2004; Svoboda et al., 2006; McDermott et al., 2009), consistent with a role of this region in social cognition and self-referential thought (Amodio and Frith, 2006; Northoff et al., 2006).

The present task-evoked dissociation of the DMN is consistent with recent fractionations based on resting-state functional connectivity analyses (Buckner et al., 2008; Meunier et al., 2009; Uddin et al., 2009; Mayer et al., 2010). For example, Uddin et al. (2009) examined the pattern of rs-fcMRI from mPFC and PCC regions and reported evidence for a dissociation along the anteroposterior axis, consistent with the present results. An alternative fractionation, however, was proposed in a recent study (Andrews-Hanna et al., 2010), likely reflecting the use of different tasks. These results indicate the difficulty of determining a single, "correct" fractionation.

The topographical reproducibility of cortical networks during either task execution or resting states (Toro et al., 2008; Smith et al., 2009) does not necessarily imply their functional homogeneity. In analogy to the existence of multiple functional networks for the processing of environmental stimuli, the apparent functional homogeneity of the DMN during environmentally directed tasks (Shulman et al., 1997; Mazoyer et al., 2001) breaks down in tasks that isolate specific internally directed processes (Hassabis et al., 2007). The deactivation of the DMN during environmentally directed tasks likely reflects the suppression of a broad differentiated set of internally directed processes that involve distinct anatomical structures. Detailed analyses will likely reveal similar fractionations within other resting-state networks (Smith et al., 2009; Nelson et al., 2010).

Finally, our results indicate a flexible or contingent relationship between networks that depends on task demands, by showing the concurrent activation of regions of the DMN and other cortical networks during goal-directed cognition. Along with other recent evidence of the flexible interaction between networks (Anticevic et al., 2010; Spreng et al., 2010), this finding does not support a fixed partitioning of the cortex between taskpositive and task-negative networks.

\section{References}

Aguirre GK, Zarahn E, D'Esposito M (1998) The inferential impact of global signal covariates in functional neuroimaging analyses. Neuroimage 8:302-306.

Amodio DM, Frith CD (2006) Meeting of minds: the medial frontal cortex and social cognition. Nat Rev Neurosci 7:268-277.

Andrews-Hanna JR, Reidler JS, Sepulcre J, Poulin R, Buckner RL (2010) Functional-anatomic fractionation of the brain's default network. Neuron 65:550-562.

Anticevic A, Repovs G, Shulman GL, Barch DM (2010) When less is more: TPJ and default network deactivation during encoding predicts working memory performance. Neuroimage 49:2638-2648.

Baddeley A (2000) The episodic buffer: a new component of working memory? Trends Cogn Sci 4:417-423. 
Badre D, Wagner AD (2007) Left ventrolateral prefrontal cortex and the cognitive control of memory. Neuropsychologia 45:2883-2901.

Boynton GM, Engel SA, Glover GH, Heeger DJ (1996) Linear systems analysis of functional magnetic resonance imaging in human V1. J Neurosci 16:4207-4221.

Buckner RL, Wheeler ME (2001) The cognitive neuroscience of remembering. Nat Rev Neurosci 2:624-634.

Buckner RL, Raichle ME, Miezin FM, Petersen SE (1996) Functional anatomic studies of memory retrieval for auditory words and visual pictures. J Neurosci 16:6219-6235.

Buckner RL, Andrews-Hanna JR, Schacter DL (2008) The brain's default network: anatomy, function, and relevance to disease. Ann N Y Acad Sci 1124:1-38.

Cabeza R, Prince SE, Daselaar SM, Greenberg DL, Budde M, Dolcos F, LaBar KS, Rubin DC (2004) Brain activity during episodic retrieval of autobiographical and laboratory events: an fMRI study using a novel photo paradigm. J Cogn Neurosci 16:1583-1594.

Courtney SM, Ungerleider LG, Keil K, Haxby JV (1997) Transient and sustained activity in a distributed neural system for human working memory. Nature 386:608-611.

Davachi L (2006) Item, context and relational episodic encoding in humans. Curr Opin Neurobiol 16:693-700.

D'Esposito M, Postle BR, Ballard D, Lease J (1999) Maintenance versus manipulation of information held in working memory: an event-related fMRI study. Brain Cogn 41:66-86.

Donaldson DI, Wheeler ME, Petersen SE (2010) Remember the source: dissociating frontal and parietal contributions to episodic memory. J Cogn Neurosci 22:377-391.

Eichenbaum H, Yonelinas AP, Ranganath C (2007) The medial temporal lobe and recognition memory. Annu Rev Neurosci 30:123-152.

Fletcher PC, Henson RN (2001) Frontal lobes and human memory: insights from functional neuroimaging. Brain 124:849-881.

Fox MD, Corbetta M, Snyder AZ, Vincent JL, Raichle ME (2006) Spontaneous neuronal activity distinguishes human dorsal and ventral attention systems. Proc Natl Acad Sci U S A 103:10046-10051.

Furman O, Dorfman N, Hasson U, Davachi L, Dudai Y (2007) They saw a movie: long-term memory for an extended audiovisual narrative. Learn Mem 14:457-467.

Goldberg I, Ullman S, Malach R (2008) Neuronal correlates of "free will" are associated with regional specialization in the human intrinsic/default network. Conscious Cogn 17:587-601.

Goldberg II, Harel M, Malach R (2006) When the brain loses its self: prefrontal inactivation during sensorimotor processing. Neuron 50:329-339.

Greicius MD, Menon V (2004) Default-mode activity during a passive sensory task: uncoupled from deactivation but impacting activation. J Cogn Neurosci 16:1484-1492.

Greicius MD, Krasnow B, Reiss AL, Menon V (2003) Functional connectivity in the resting brain: a network analysis of the default mode hypothesis. Proc Natl Acad Sci U S A 100:253-258.

Hassabis D, Kumaran D, Maguire EA (2007) Using imagination to understand the neural basis of episodic memory. J Neurosci 27:14365-14374.

He BJ, Snyder AZ, Vincent JL, Epstein A, Shulman GL, Corbetta M (2007) Breakdown of functional connectivity in frontoparietal networks underlies behavioral deficits in spatial neglect. Neuron 53:905-918.

Henson RN, Rugg MD, Shallice T, Josephs O, Dolan RJ (1999) Recollection and familiarity in recognition memory: an event-related functional magnetic resonance imaging study. J Neurosci 19:3962-3972.

Iacoboni M, Lieberman MD, Knowlton BJ, Molnar-Szakacs I, Moritz M, Throop CJ, Fiske AP (2004) Watching social interactions produces dorsomedial prefrontal and medial parietal BOLD fMRI signal increases compared to a resting baseline. Neuroimage 21:1167-1173.

Kahn I, Davachi L, Wagner AD (2004) Functional-neuroanatomic correlates of recollection: implications for models of recognition memory. J Neurosci 24:4172-4180.

Mayer JS, Roebroeck A, Maurer K, Linden DE (2010) Specialization in the default mode: task-induced brain deactivations dissociate between visual working memory and attention. Hum Brain Mapp 31:126-139.

Mazoyer B, Zago L, Mellet E, Bricogne S, Etard O, Houdé O, Crivello F, Joliot M, Petit L, Tzourio-Mazoyer N (2001) Cortical networks for working memory and executive functions sustain the conscious resting state in man. Brain Res Bull 54:287-298.

McDermott KB, Szpunar KK, Christ SE (2009) Laboratory-based and autobiographical retrieval tasks differ substantially in their neural substrates. Neuropsychologia 47:2290-2298.

Mecklinger A (2010) The control of long-term memory: brain systems and cognitive processes. Neurosci Biobehav Rev 34:1055-1065.

Mendelsohn A, Furman O, Dudai Y (2010) Signatures of memory: brain coactivations during retrieval distinguish correct from incorrect recollection. Front Behav Neurosci 4:18.

Meunier D, Lambiotte R, Fornito A, Ersche KD, Bullmore ET (2009) Hierarchical modularity in human brain functional networks. Front Neuroinformatics 3:37.

Moscovitch M, Winocur G (1995) Frontal lobes, memory, and aging. Ann N Y Acad Sci 769:119-150.

Nakayama K, Silverman GH (1986) Serial and parallel processing of visual feature conjunctions. Nature 320:264-265.

Nelson SM, Cohen AL, Power JD, Wig GS, Miezin FM, Wheeler ME, Velanova K, Donaldson DI, Phillips JS, Schlaggar BL, Petersen SE (2010) A parcellation scheme for human left lateral parietal cortex. Neuron 67:156-170.

Northoff G, Heinzel A, de Greck M, Bermpohl F, Dobrowolny H, Panksepp J (2006) Self-referential processing in our brain-a meta-analysis of imaging studies on the self. Neuroimage 31:440-457.

Ochsner KN, Knierim K, Ludlow DH, Hanelin J, Ramachandran T, Glover G, Mackey SC (2004) Reflecting upon feelings: an fMRI study of neural systems supporting the attribution of emotion to self and other. J Cogn Neurosci 16:1746-1772.

Ollinger JM, Shulman GL, Corbetta M (2001a) Separating processes within a trial in event-related functional MRI: I. The method. Neuroimage 13:210-217.

Ollinger JM, Corbetta M, Shulman GL (2001b) Separating processes within a trial in event-related functional MRI: II. Analysis. Neuroimage 13:218-229.

Raichle ME, MacLeod AM, Snyder AZ, Powers WJ, Gusnard DA, Shulman GL (2001) A default mode of brain function. Proc Natl Acad Sci U S A 98:676-682.

Rugg MD, Otten LJ, Henson RN (2002) The neural basis of episodic memory: evidence from functional neuroimaging. Philos Trans R Soc Lond B Biol Sci 357:1097-1110.

Rushworth MF, Behrens TE, Johansen-Berg H (2006) Connection patterns distinguish 3 regions of human parietal cortex. Cereb Cortex 16:1418-1430.

Sestieri C, Shulman GL, Corbetta M (2010) Attention to memory and the environment: functional specialization and dynamic competition in human posterior parietal cortex. J Neurosci 30:8445-8456.

Shulman GL, Fiez JA, Corbetta M, Buckner RL, Miezin FM, Raichle ME, Petersen SE (1997) Common blood flow changes across visual tasks: II. Decreases in cerebral cortex. J Cogn Neurosci 9:648-663.

Shulman GL, Ollinger JM, Akbudak E, Conturo TE, Snyder AZ, Petersen SE, Corbetta M (1999) Areas involved in encoding and applying directional expectations to moving objects. J Neurosci 19:9480-9496.

Shulman GL, McAvoy MP, Cowan MC, Astafiev SV, Tansy AP, d'Avossa G, Corbetta M (2003) Quantitative analysis of attention and detection signals during visual search. J Neurophysiol 90:3384-3397.

Shulman GL, Astafiev SV, McAvoy MP, d'Avossa G, Corbetta M (2007) Right TPJ deactivation during visual search: functional significance and support for a filter hypothesis. Cereb Cortex 17:2625-2633.

Smith SM, Fox PT, Miller KL, Glahn DC, Fox PM, Mackay CE, Filippini N, Watkins KE, Toro R, Laird AR, Beckmann CF (2009) Correspondence of the brain's functional architecture during activation and rest. Proc Natl Acad Sci U S A 106:13040-13045.

Spreng RN, Stevens WD, Chamberlain JP, Gilmore AW, Schacter DL (2010) Default network activity, coupled with the frontoparietal control network, supports goal-directed cognition. Neuroimage 53:303-317.

Svoboda E, McKinnon MC, Levine B (2006) The functional neuroanatomy of autobiographical memory: a meta-analysis. Neuropsychologia 44:2189-2208.

Talairach J, Tournoux P (1988) Co-planar stereotaxic atlas of the human brain. New York: Thieme Medical Publishers.

Todd JJ, Marois R (2004) Capacity limit of visual short-term memory in human posterior parietal cortex. Nature 428:751-754. 
Toro R, Fox PT, Paus T (2008) Functional coactivation map of the human brain. Cereb Cortex 18:2553-2559.

Treisman A (1988) Features and objects: the fourteenth Bartlett memorial lecture. Q J Exp Psychol A 40:201-237.

Uddin LQ, Kelly AM, Biswal BB, Xavier Castellanos F, Milham MP (2009) Functional connectivity of default mode network components: correlation, anticorrelation, and causality. Hum Brain Mapp 30:625-637.

Uddin LQ, Supekar K, Amin H, Rykhlevskaia E, Nguyen DA, Greicius MD, Menon V (2010) Dissociable connectivity within human angular gyrus and intraparietal sulcus: evidence from functional and structural connectivity. Cereb Cortex 20:2636-2646.

Van Essen DC (2005) A population-average, landmark- and surface-based (PALS) atlas of human cerebral cortex. Neuroimage 28:635-662.

Vilberg KL, Rugg MD (2007) Dissociation of the neural correlates of recog- nition memory according to familiarity, recollection, and amount of recollected information. Neuropsychologia 45:2216-2225.

Vilberg KL, Rugg MD (2008) Memory retrieval and the parietal cortex: a review of evidence from a dual-process perspective. Neuropsychologia 46:1787-1799.

Vincent JL, Snyder AZ, Fox MD, Shannon BJ, Andrews JR, Raichle ME, Buckner RL (2006) Coherent spontaneous activity identifies a hippocampal-parietal memory network. J Neurophysiol 96:35173531.

Wagner AD, Shannon BJ, Kahn I, Buckner RL (2005) Parietal lobe contributions to episodic memory retrieval. Trends Cogn Sci 9:445-453.

Yonelinas AP, Otten LJ, Shaw KN, Rugg MD (2005) Separating the brain regions involved in recollection and familiarity in recognition memory. J Neurosci 25:3002-3008. 